\title{
Technical methods of emissivity correction in thermography
}

by WIECEK B. * and PACHOLIK J. *

* Technical University of Lodz, Institute of Electronics, 18-22 Stefanowskiego, str., 90924 Lodz, Poland

\begin{abstract}
This article presents some chosen methods of temperature correction while the emissivity factor of the investigated object is nonuniform on its surface. An approach of application of reflection method (RM) for emissivity factor evaluation is emphasised in comparison with others based on alternate temperature measurement or covering object surface by thin layer with known emissivity factor. The practical possibilities as well as the limits of these methods are discussed. The examples of temperature map correction are presented.
\end{abstract}

\section{Introduction}

One of the main problems, still existing in thermography is the temperature correction by the use of emissivity factor, which is typically nonuniform on the object surface. In variety of applications where there are plenty of elements with different emissivity factors, the direct thermographic measurement can lead to wrong results, with temperature errors higher sometimes than $100 \%$ [4]. Hence, the emissivity factor evaluation is demanded in thermography, and actually novel attempts to implement different methods is still being taken $[3,4]$. In order to get satisfying results two separate tasks should be technically solved:

- evaluation of emissivity factor for given details of the object,

- automatic correction of the thermal image in the computer.

The first task of emissivity evaluation can be performed by some traditional methods, not convenient in every application, especially when the correction has to be done whilo the object is in its normal working conditions (e.g. electrical machine under the load). We recommend non-invasive method based reflection radiation measurement - reflection method (RM).

The second problem has been solved by using computer-based thermographic system built up in the Institute of Electronics, Technical University of Lodz [2]. Software working in MS-WINDOWS environment allows to choose given detail of the image on the screen and to enter the value of emissivity factor for this detail. Temperature correction is dono. automatically.

One of the previous methods of emissivity equalising is based on covering surface by thin layer with high emissivity factor. Typically, one can use black paint with tho emissivity factor $\varepsilon=0.95-1$ to cover measured object. In Fig. 1 . a working electronic circuit (power converter) is presented before and after painting. The measurements have been performed by HUGHES TVS-40TE Thermal Video System and PC-based digitizer. This very simple method has some limits of its application:

- covering even by very thin layer changes slightly the heat transfer in the investigated body,

- in electrical, high voltage environment it is rather dangerous to use it while the electrical system is working, because of the elements isolation. 
http://dx.doi.org/10.21611/qirt.1994.021

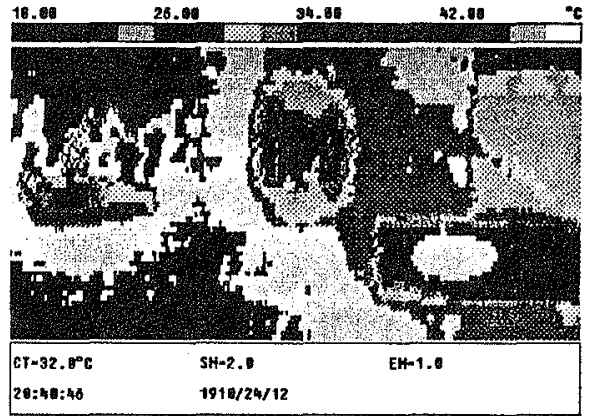

a)

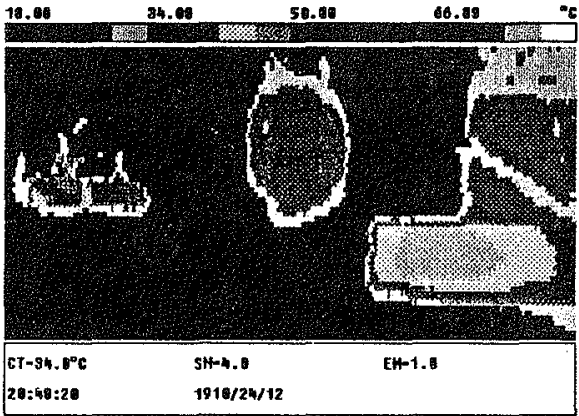

b)

Fig. 1. Temperature map of the converter (from the left side : 2 diodes placed on the radiators, transformer and resistive load) a) before covering with the black paint $\varepsilon \sim 1.0$, b) after painting

The other approach to emissivity evaluation is based on colorimetric surveys. So far, we know the object temperature and the radiation level by thermographic measurement, the simple formulas give the emissivity factor estimation. If one considers the ambient radiation, the following expressions are valid:

$\varepsilon T^{4}+T_{a}^{4}=T_{m}^{4} \quad \varepsilon=\frac{T_{m}^{4}-T_{a}^{4}}{T^{4}}$

where: $T, T_{a}$ and $T_{m}$ denote object, ambient and measured by thermal camera temperature (substitute temperature), respectively.

Using measured $\varepsilon$ one can automatically correct temperature maps, that can be seen in Fig.9 (using RM method). We should underline that this method is valid as far as the emittance does not depend on the temperature and wavelength, what is only true in the narrow temperature range. Practically, this method has been verified successfully in electronics, where the temperature variation does not exceed 100-200K.

Additionally, one should indicate that for the reason of some measurement disturbances [5], the emissivity factor and, in consequence temperature is multivalent. In many practical cases the exact value of emissivity is not required, and one can be satisfied by getting a range for the estimated value of $\varepsilon$ (confidence interval).

\section{Reflection methods}

The behaviour of electromagnetic waves on the border of two environments can be described as:

$$
\alpha+\beta+\tau=1 .
$$

where: $\alpha$ - absorptivity, $\beta$ - reflectivity and $\tau$ - transparency.

For the objects with their transparency equal to zero (solid materials) and if radiation properties are independent of the wavelength one can set:

$$
\varepsilon=1-\beta \text {. }
$$

So, for the objects whose temperature is equal to the ambient temperature, it is possible to evaluate the emissivity factor on the basis of measurements of incident and reflected energy. 
http://dx.doi.org/10.21611/qirt.1994.021

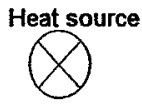

infrared camera
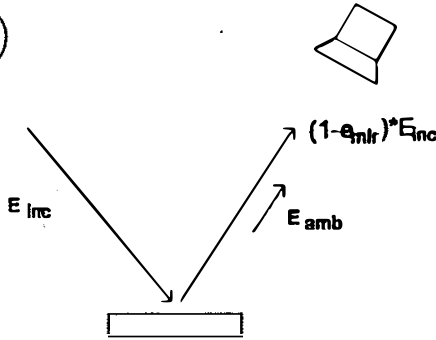

infrared mirror

Fig 2. - Incident flux measurement, $\varepsilon_{\text {mir }}$ - emissivity of the infrared mirror (close to zerol, Einc - incident energy density

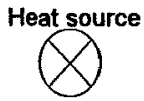

infrared camera

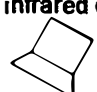

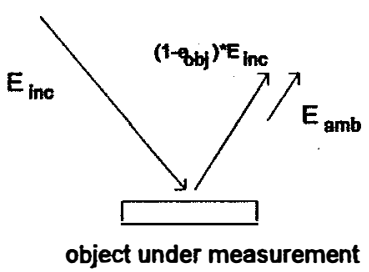

Fig. 3. - Reflected flux measurement, $\varepsilon_{\text {obj }}$ - emissivity of the object, $E_{\text {amb }}$ - energy density emitted by thermal environment

In order to evaluate hemispherical emissivity (not the spectral one) the heat source has to emit the waves that totally cover spectral range of the infrared detector (for

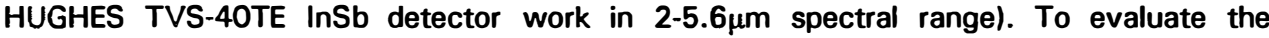
incident flux, the infrared mirror (highly polished copper plate) with reflection factor about 0.98 , independent of the wavelength in the spectral range mentioned above, has been used. The set of metal bodies (Al, Fe) with different surface states has been prepared to work out the proper measurement method. The emissivity factor for each body was evaluated by using calorimetric method. Obtained values of emissivity varies from 0.25 to 0.45 .

2.1 Emissivity factor evaluation based on the measurements of the fluxes for the mirror angles.

The first measurements of the emissivity factor have been based on the following assumption:

because the reflection characteristic for metals is very selective (very small deviation from the mirror angle causes very rapid decrease of the reflectivity - low diffusionl, the main part of the flux is reflected in the mirror angle and it is possible to evaluate the emissivity factor on the basis of measurements of reflected fluxes for given body and infrared mirror.

When the emissivity parameter set in the infrared camera is equal to 1.0 , measured temperature evaluates incident flux:

$$
\sigma T_{C u}^{4}=\beta_{C u}{ }^{*} E_{i n c}+E_{a m b}
$$

where: $E_{a m b}=\sigma T_{a m b}{ }^{4}, \beta C u$ - reflectivity of copper mirror $(\approx 1), E_{i n c}$ - incident flux, $\mathrm{T}_{\mathrm{Cu}}{ }^{-}$ substitute temperature of the copper mirror and $\mathrm{T}_{\mathrm{amb}}$ - ambient temperature.

In the same way the reflected flux has to be evaluated as:

$$
\sigma T_{m}^{4}=(1-\varepsilon)^{*} E_{i n c}+E_{a m b}
$$

where: $T_{m}$-substitute temperature of the detail under measurement.

Hence, the value of emissivity factor can be calculated as:

$$
\varepsilon=1-\left(T_{m}^{4}-T_{a n b}^{4}\right) /\left(T_{C u}^{4}-T_{a m b}^{4}\right)
$$


In some practical cases one can get very high values of emissivity factor $(\approx 0.9)$ by using (6), that is simply incorrect. It means, that generally only a part of energy is reflected in the mirror angle. The difference between obtained values and results presented in the literature are caused first of all by different surface conditions of the measured samples [1].

\subsection{Evaluation of the emissivity by the use of correlation}

As it has been shown in the previous chapter, the assumptions on the mirror reflection even in the case of metal bodies are not always valid. So, the diffuse reflection also has to be taken into account. The shape of the reflectivity characteristic for the real objects considering both mirror and diffusion reflection is shown in [1]. This characteristic depends not only upon the spectral properties of the incident flux but upon its incident angle $\alpha$ as well. It is noticeable that, the larger $\alpha$, the higher maximum of this characteristic. The more "mirrored" reflection, the more selected reflection characteristic, and in contrast the more "diffused" reflection the flatter characteristic. So the correlation between the value of the flux reflected in the mirror angle and a certain parameter describing the slope of the characteristic is expected.

As this parameter, the factor $\delta$, that is the ratio of the reflected flux for the angle $\beta$ deviated from the mirror angle and the flux reflected in the mirror angle $\beta_{\text {mir, }}$, has been introduced. For the given detail of the body three measurements of the reflected flux has to be done:

- for infrared mirror and mirror angle

- for given object and mirror angle

- for given object and the angle deviated from the mirror angle

The relative value $K$ of the energy reflected in the mirror angle takes a form:

$$
K=\left(\sigma T_{m}^{4}-E_{a m b}\right) /\left(\sigma T_{C u}^{4}-E_{a m b}\right)
$$

where: $\quad E_{a m b}=\sigma T_{a m b}^{4}$.

Hence:

$$
K=\left(T_{m}^{4}-T_{a m b}^{4}\right) /\left(T_{C u}^{4}-T_{a m b}^{4}\right)
$$

The parameter $\delta$ of the shape of the reflection characteristic is defined as:

$$
\delta=\left(T_{\beta}^{4}-T_{a m b}^{4}\right) /\left(T_{m}^{4}-T_{a m b}^{4}\right)
$$

where: $T_{\beta}, T_{m}, T_{a m b}, T_{C u}$ mean: substitute temperature measured in the case of deviation angle $\beta$, temperature measured in the mirror angle, ambient temperature and substitute temperature for the infrared mirror defining temperature of the heat source, respectively.

Real emissivity of the samples has been evaluated by calorimetric method and the rectangles in Fig.4 denote the range of the results considering precision of the measurements and environment noise.

The majority of samples well tiles the correlation curve which is highly non-linear but still monotonous. Some of the results differ significantly from obtained characteristic. It is caused by the anisotropy of the reflection that exists in many practical cases, what the method mentioned above does not take into account. In order to take this phenomenon into account the measurements in different directions have to be made. 


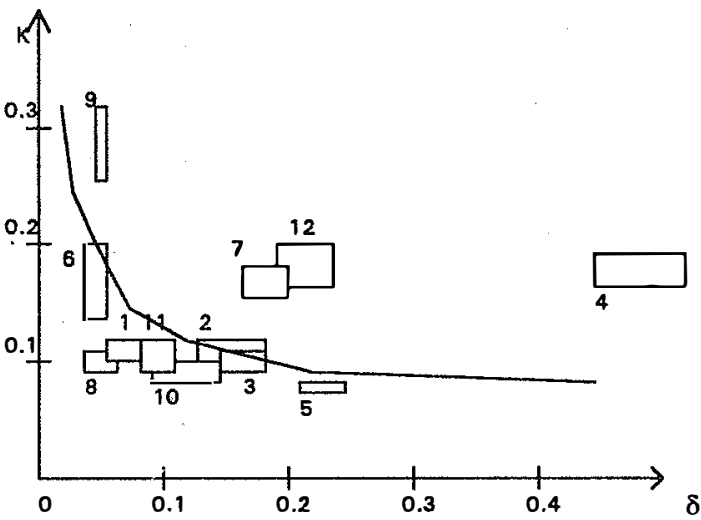

Fig. 4. Correlation curve of the reflected flux in the mirror angle arid the parameter $\delta$ defining the shape of the characteristic of the reflectivity, samples: 1 -5 Al $\varepsilon=0.31-0.35$; $6-\mathrm{Fe}$ : $=0.44-0.51,7-8 \mathrm{Fe} \varepsilon=0.36-0.42,9 \mathrm{Al} \varepsilon$ $=0.45-0.49,10$ Al $\varepsilon=0.4-0.45,11 \mathrm{Al} \mathrm{s}$ $=0.32-0.38,12 \mathrm{Fe} \varepsilon=0.44-0.5$

\subsection{Evaluation of the emissivity factor considering anisotropy of the reflection.}

The effect of reflection anisotropy has been found for the samples with distinct texture on the surface. If one of the directions on the surface of the sample is marked out (e.g.: in the mat process), the reflectivity characteristic in that direction will differ significantly from the characteristic measured in the perpendicular one (Fig.5). The value of the reflected energy $E$ is expressed in the conventional thermal units described as: $E[J T]=\left(T^{4}-T_{a m b}^{4}\right) / 10^{8}, \Delta \phi$ means the deviation from the mirror angle.

Total value of the reflected energy is evaluated as a volume of the solid limited by the shapes of the reflectivity characteristics for all directions of observation. In practical cases it is rather difficult to measure total spherical characteristic of the reflectivity, and so a simplification has to be made. In order to calculate the volume of such a solid tho following assumption is set:

- the cross-section of this solid is in the shape of ellipse whose semiaxes lies in tha privileged direction and the direction perpendicular to it.

In order to check the correctness of this assumption the cross-sections of the spherical reflectivity characteristic have been measured (Fig.6). The cross-sections shown in the Fig. 6 have been obtained from the characteristics of the reflectivity measured in different angle of observation $\alpha$ (alfa1, alfa2, alfa3) within the range $0-90^{\circ}$. The change of the observation angle has been obtained by rotating the sample about given angle $\alpha$. For each rotation angle the characteristic of the reflectivity has been measured $E[J T]=f(\Delta \phi)$.

The assumption of the ellipsoidal shape of the cross-section is not valid in the case of the samples with high anisotropy. It leads to the errors in calculations of the volume of tho solid, which is the measure of the total reflected energy. Additional problem is how to choose the directions of measurements properly.

In order to check correctness of the proposed method some measurements for different samples have been made. The results have been compared with ones obtained by using calorimetric method. Results obtained by RM have been in the same range as in this case of calorimetric method (Fig. 9). Honestly, it has to be said that the accuracy of RM sometime is rather poor le.g.: in the case of the sample with low emissivity $0.31-0.36$ measured by calorimetric method, the results obtained by reflection method has beon about 0.3-0.4, sometimes even 0.2-0.4).

\section{Conclusions}

In order to verify practically the RM, a circuit consisting of a pair of power transistoris (in metal case TO3) placed on the black painted radiator, has been chosen. To compari the results, the measurements in the same conditions but after covering all the elements 
http://dx.doi.org/10.21611/qirt.1994.021

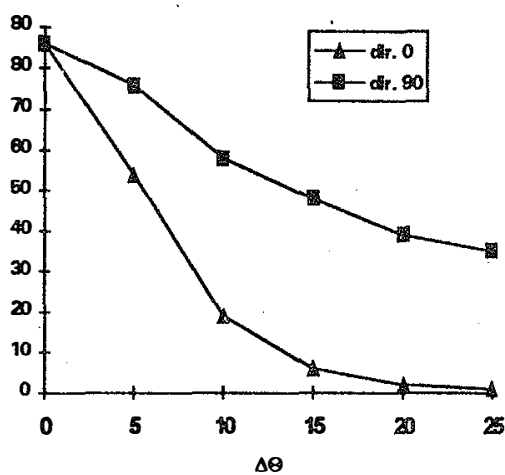

Fig.5. The reflectivity characteristics for one of the samples with anisotropy on the surface for two perpendicular directions of the observations.

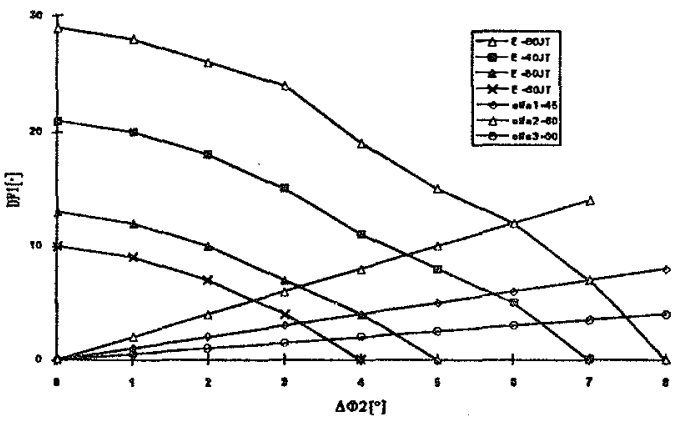

Fig.6. The cross-sections of the spherical reflectivity characteristic for the sample with anisotropy on the surface, $\Delta \Phi_{1}, \Delta \Phi_{2}$ means the angles between the camera axis and normal to the object in given point for two perpendicular directions.

with black paint $(0.9)$ are performed. Fig.7a shows the temperature map in the direct sight of the infrared camera.

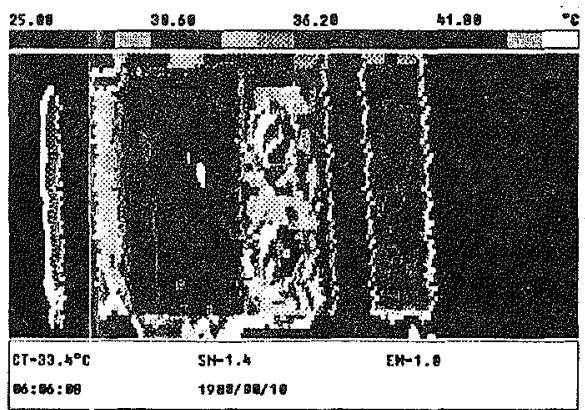

a)

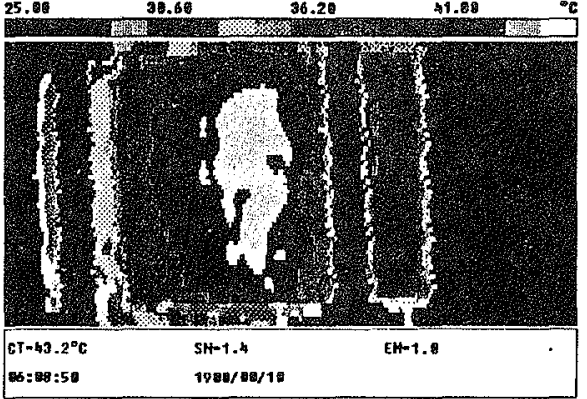

b)

Fig. 7. Temperature map of the power transistors in the end stage of power supply unit (10V. 10A), a) before covering with the black paint $\varepsilon \sim 1.0$, b) after painting

The situation seems to be paradoxical - the heat source has got lower temperature than the cooling environment. Real temperature map obtained after painting the object is shown in Fig. $7 b$.

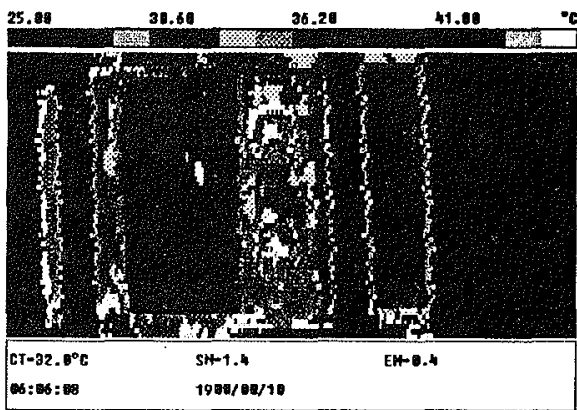

Fig. 8. Temperature map after $\varepsilon$ correction for upper side of the transistors, $\varepsilon=\mathbf{0 . 3 5}$
On the basis of the measurements of the emissivity using RM, the correction of the temperature map has been done. One can say that the evaluation of the emissivity in the range 0.3-0.4 gives significant improvement of the temperature measurements. The RM gives good results in the laboratory, when the measurements can be done precisely and with non portable equipment. In the last Fig. 9 corrected thermal maps for power converter mentioned in chapter 1 are presented. There is a quite good accordance of the result of $R M$ in 
http://dx.doi.org/10.21611/qirt.1994.021

comparison with traditional method of covering by thin layer (Fig.1b).
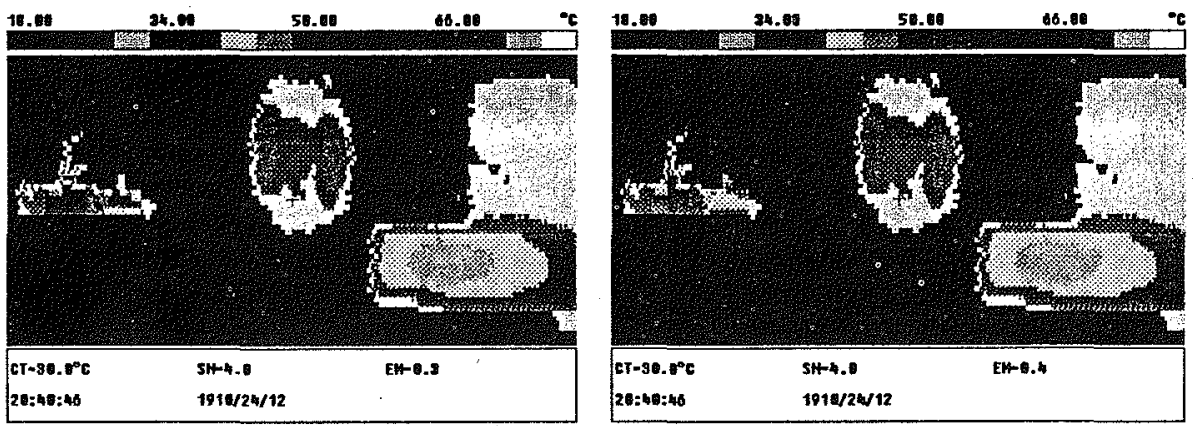

Fig. 9. - Temperature map of the converter after correction of emissivity factor for converter presented above - RM method, a) for $\varepsilon=0.3$, b) for $\varepsilon=0.4$

It has to be said that there are some problems that limit the range of applications:

- difficulty to find the mirror angle very precisely.

- precision of reflected energy measurement with high anisotropy,

- the error resulting from calculations is rather high lin the case of the samples with very low emissivities it can even rise over $50 \%$ l,

\section{REFERENCES}

[1] SALA (A.).- Radiation heat transfer (in Polish), WNT. Warszawa 1982.

[2] WIECEK (B.), GRECKI (M.), PACHOLIK (J.). - Computer -based thermographic system, Proc. Sem. Quantitative Infrared Thermography, 1992, pp. 57-60

[3] WIECEK (B.), GRECKI (M.), PACHOLIK (J.). - Themal measurement of power electronic devices using thermographic system. Proc. Sem., 1992, pp. 291-295

[4] WIECEK (B.), GRECKI (M.), PACHOLIK (J.). - Emissivity Factor Evaluation Using the Reflection Method for Application of IR System in Electronics. Proc. Conf. Advanced Infrared Technology and Application, Casa Malaparte, Capri, 1993, pp. 291-303

[5] WIECEK (B.), GRECKI (M.), PACHOLIK (J.). - Extention of Thermal Image System by Visual Camera Interface. Proc. Conf. Advanced Infrared Technology and Application, Casa Malaparte, Capri, 1993, pp. 79-89 